\title{
Fourier-spectral-method implementation of deformation in the phase-field crystal model
}

\author{
Nirand Pisutha-Arnond ${ }^{1, *}$ \\ ${ }^{1}$ Department of Industrial Engineering, Faculty of Engineering, King Mongkut's Institute of Technology Ladkrabang, Bangkok 10520, \\ Thailand
}

\begin{abstract}
The phase-field crystal (PFC) method is a promising computational model with atomistic resolution and diffusive time scale. In this work, the Fourier-spectral-method (FSM) scheme was developed for evaluating the PFC free energy of a system subjected homogeneous deformation. This scheme addresses the complication where, in numerical implementation of FSM using discrete Fourier transform (DFT), the discretized data may no longer lie along the directions of the Cartesian basis due to deformation. In this scheme, the real-space coordinate transformation is employed so that the (continuous) Fourier transform is performed on the function of the undeformed coordinates. This transformation allows straightforward DFT implementation because the sampling at the undeformed configuration is unaffected by the deformation. This scheme is also shown to be applicable to both the original PFC model and a "CDFT"-type PFC model containing a two-body correlation function.
\end{abstract}

\section{Introduction}

The phase-field crystal (PFC) model [1] is a promising model for simulating atomistic phenomena on diffusive time scale. The PFC model is based on a free energy functional that can be minimized by uniform or periodic order-parameter functions. The latter, in particular, can be used to represent atomic density in a crystal lattice, which gives the PFC model an atomic resolution. The timeevolution of the order parameter is driven by dissipative dynamics which leads to the (diffusive) time scale that is not bounded by prohibitively small atomic-vibration time scale. These features make the PFC model appealing for simulating nano- to meso-scale materials phenomena over the time scale that is relevant to experimental observation. (The interested reader may consult Ref. [2] and the references therein for comprehensive information of the PFC model.)

Since the order-parameter profiles in the PFC model are typically smooth, periodic, and contained in a geometrically simple computational domain, the Fourier spectral method (FSM) provides an efficient and accurate algorithm for evaluating the PFC free energy and solving the dynamic equations. The reason is that, in Fourier space, spatial derivative becomes multiplication, which can be computed efficiently. Also, the FSM is a global approach which yields high accuracy (known as spectral accuracy) unmatched by local methods such as finite difference. These advantages make the FSM a robust numerical method for PFC calculations.

In numerical implementation of FSM, the discrete Fourier transform (DFT) is used to approximate the con-

*e-mail: nirand.pi@kmitl.ac.th tinuous Fourier transform. For the DFT, it is assumed that the discrete data are sampled along the directions of the Cartesian basis; in two dimensions, for example, one can visualize the data forming a rectangular grid. However, the complication arises when the system is deformed and the grid is no longer rectangular. How does one implement the DFT, and FSM in general, in this situation?

In this work, I presented the FSM scheme for calculating the PFC free energy of a system subjected to homogeneous deformation. This scheme utilizes real-space coordinate transformation so that the (continuous) Fourier transform is performed on the function of the undeformed coordinates and the effect of the deformation instead manifests in the transformation of the Fourier wave vector. The advantage of this scheme is the straightforward DFT implementation because the function of the undeformed configuration and its discretized data are not affected by the deformation. This technique is, in part, similar to that used in imaging processing to obtain the Fourier representation of affine-transformed image data [3]. However, in the context of the PFC model, the spatial derivative needs to be included in the derivation and the application of this scheme to the PFC free energy that contains a two-body correlation function (referred to as the "CDFT"-type PFC [4]) is discussed. The scheme developed in this work not only applies to the PFC model, but also to other models that can take advantages of the efficiency and accuracy of the FSM.

The paper is organized as follows. In Section 2, the background information on the PFC model, the (continuous) Fourier transform, and the measure of deformation are reviewed. The FSM scheme to evaluate the PFC free energy of the deformed system is presented in Section 3 
and the extension of the scheme to the CDFT-type PFC is presented in Section 4. The numerical verification of the proposed scheme is shown in Section 5, followed by the summary in Section 6.

\section{Background}

\subsection{Phase-Field Crystal Model}

The original form of the PFC free energy functional, $F_{\mathrm{pfc}}$, is $[1,5]$

$$
\begin{gathered}
F_{\mathrm{pfc}}=\int_{V} f_{\mathrm{pfc}}(\mathbf{x}) d \mathbf{x}, \\
f_{\mathrm{pfc}}(\mathbf{x})=\frac{\phi(\mathbf{x})}{2}\left[-\epsilon+\left(\nabla_{\mathbf{x}}^{2}+1\right)^{2}\right] \phi(\mathbf{x})+\frac{\phi(\mathbf{x})^{4}}{4},
\end{gathered}
$$

where the (field) variable $f_{\text {pfc }}$ is the free energy density (FED), $\phi(\mathbf{x})$ is the order parameter representing the atomic density field, $\epsilon$ is the model parameter, and $V$ is the volume of the system. The variable, $\mathbf{x}=x_{i} \mathbf{e}_{i}$ is a position vector expressed in terms of the Cartesian basis vectors, $\mathbf{e}_{i}$, where the Einstein summation convention is used throughout the paper and the subscript $i$ varies from 1 to 3 in three dimensions. The operatior $\nabla_{\mathbf{x}}^{2}$ is the Laplacian written in terms of $\mathbf{x}$, or $\nabla_{\mathbf{x}}^{2}=\partial^{2} / \partial x_{i} \partial x_{i}$.

\subsection{Fourier Transform}

The (continuous) Fourier transform used in this work is defined as

$$
\hat{h}(\mathbf{s})=\int_{-\infty}^{\infty} h(\mathbf{x}) e^{-i \mathbf{s} \cdot \mathbf{x}} d \mathbf{x},
$$

where $\mathbf{s}$ is the Fourier wave vector and $\hat{h}(\mathbf{s})$ is the Fourier representation of the function $h(\mathbf{x})$. The corresponding inverse Fourier transform is defined as

$$
h(\mathbf{x})=\frac{1}{(2 \pi)^{d}} \int_{-\infty}^{\infty} h(\mathbf{s}) e^{i \mathbf{s} \cdot \mathbf{x}} d \mathbf{s},
$$

where $d$ is the dimensionality of the problem.

\subsection{Measure of Deformation}

To represent deformation, one can define $\mathbf{R}=R_{i} \mathbf{e}_{i}$ and $\mathbf{r}=r_{i} \mathbf{e}_{i}$ to be the position vectors that are attached to material points in the undeformed and deformed configurations, respectively. A transformation that maps $\mathbf{R}$ to $\mathbf{r}$ is described by a deformation-gradient tensor, $\alpha$, whose elements are defined as $\alpha_{i j}=\partial r_{i} / \partial R_{j}$. For homogeneous deformation, $\alpha$ is spatially independent and one can write the transformation in tensor notations: $\mathbf{r}=\boldsymbol{\alpha} \mathbf{R}$ and $\mathbf{R}=\boldsymbol{\alpha}^{-1} \mathbf{r}$. Also, $J=\operatorname{det}(\boldsymbol{\alpha})$ is the volume ratio $V / \mathcal{V}$, where $V$ and $\mathcal{V}$ is the deformed and undeforemd volumes respectively.

The deformation type used for numerical verification is characterized by $r_{1}=\eta R_{1}-\xi R_{2}, r_{2}=\eta R_{2}$, and $r_{3}=\eta R_{3}$, where $\eta=1+\xi$. This gives $J=\eta^{3}$ and

$$
\boldsymbol{\alpha}=\left[\begin{array}{ccc}
\eta & -\xi & 0 \\
0 & \eta & 0 \\
0 & 0 & \eta
\end{array}\right], \quad \boldsymbol{\alpha}^{-1}=\frac{1}{\eta}\left[\begin{array}{ccc}
1 & \xi / \eta & 0 \\
0 & 1 & 0 \\
0 & 0 & 1
\end{array}\right] .
$$

\section{FSM Scheme to Evaluate PFC Free Energy of a Deformed System}

To calculate the free energy of the deformed system [6], one expresses the order parameter with the coordinates $\mathbf{R}(\mathbf{r})=\boldsymbol{\alpha}^{-1} \mathbf{r}$, which yields $\phi(\mathbf{R}(\mathbf{r}))$. This functional representation relates the functional values in the deformed configuration to those in the undeformed configuration. Writing the integral (Eq. (1)) and the Laplacian (Eq. (2)) in terms of $\mathbf{r}$, one arrives at

$$
\begin{gathered}
F_{\mathrm{def}}=\int_{V} f_{\mathrm{def}}(\mathbf{r}) d \mathbf{r}=J \int_{\mathcal{V}} f_{\mathrm{def}}(\mathbf{r}(\mathbf{R})) d \mathbf{R} \\
f_{\mathrm{def}}(\mathbf{r})=\frac{\phi(\mathbf{R}(\mathbf{r}))}{2}\left[-\epsilon+\left(\nabla_{\mathbf{r}}^{2}+1\right)^{2}\right] \phi(\mathbf{R}(\mathbf{r}))+\frac{\phi(\mathbf{R}(\mathbf{r}))^{4}}{4},
\end{gathered}
$$

where $\nabla_{\mathbf{r}}^{2}=\partial^{2} / \partial r_{i} \partial r_{i}$. Since the effective use of the FSM is on evaluation of the terms in Eq. (7) with Laplacian (and the higher order Laplacian), I will only consider a simplified example of

$$
f_{\mathrm{ex} 1}(\mathbf{r})=\nabla_{\mathbf{r}}^{2} \phi(\mathbf{R}(\mathbf{r}))
$$

to demonstrate the FSM scheme.

\subsection{Forward Transformation}

By using Eq. (3), the continuous Fourier transform of $f_{\text {ex } 1}(\mathbf{r})$ is written as

$$
\hat{f}_{\mathrm{ex} 1}(\mathbf{w})=\int_{-\infty}^{\infty} f_{\mathrm{ex} 1}(\mathbf{r}) e^{-i \mathbf{w} \cdot \mathbf{r}} d \mathbf{r}=\int_{-\infty}^{\infty} \nabla_{\mathbf{r}}^{2} \phi(\mathbf{R}(\mathbf{r})) e^{-i \mathbf{w} \cdot \mathbf{r}} d \mathbf{r}
$$

The goal here is to transform the coordinates from $\mathbf{r}$ to $\mathbf{R}$. First, the volume integration is rewritten as

$$
\int_{-\infty}^{\infty} d \mathbf{r}=J \int_{-\infty}^{\infty} d \mathbf{R}
$$

Second, the Fourier basis is re-expressed as

$$
\begin{aligned}
\exp (-i \mathbf{w} \cdot \mathbf{r}) & =\exp [-i \mathbf{w} \cdot(\boldsymbol{\alpha} \mathbf{R})] \\
& =\exp \left[-i\left(\boldsymbol{\alpha}^{T} \mathbf{w}\right) \cdot \mathbf{R}\right] \\
& =\exp [-i \mathbf{W} \cdot \mathbf{R}],
\end{aligned}
$$

where $\mathbf{W}=\boldsymbol{\alpha}^{T} \mathbf{w}$ is the transformed Fourier wave vector. This transformation is how the effect of deformation is "carried" into the Fourier space. Finally, the Laplacian can be transformed using the chain rule $\partial / \partial r_{m}=$ $\left(\partial R_{i} / \partial r_{m}\right) \partial / \partial R_{i}=\alpha_{i m}^{-1} \partial / \partial R_{i}$. This yields

$$
\nabla_{\mathbf{r}}^{2}=\alpha_{i m}^{-1} \alpha_{j m}^{-1} \frac{\partial}{\partial R_{i}} \frac{\partial}{\partial R_{j}} \equiv \nabla_{\mathbf{r}(\mathbf{R})}^{2} .
$$

Using Eqs. (10), (11), and (12), Eq. (9) can be rewritten as

$$
\hat{f}_{\mathrm{ex} 1}(\mathbf{w}(\mathbf{W}))=J \int_{-\infty}^{\infty}\left(\alpha_{i m}^{-1} \alpha_{j m}^{-1} \frac{\partial}{\partial R_{i}} \frac{\partial}{\partial R_{j}}\right) \phi(\mathbf{R}) e^{-i \mathbf{W} \cdot \mathbf{R}} d \mathbf{R},
$$


where $\mathbf{w}(\mathbf{W}) \equiv \boldsymbol{\alpha}^{-T} \mathbf{W}$. Next, the partial derivative $\partial / \partial R_{m}$ can be eliminated by performing integration by parts; as it turns out, each operation $\partial / \partial R_{m}$ results in a factor of $-i W_{m}$. Therefore, the above equation can be expressed as

$$
\begin{aligned}
\hat{f}_{\mathrm{ex} 1}(\mathbf{w}(\mathbf{W})) & =J\left(-\alpha_{i m}^{-1} \alpha_{j m}^{-1} W_{i} W_{j} \int_{-\infty}^{\infty} \phi(\mathbf{R}) e^{-i \mathbf{W} \cdot \mathbf{R}} d \mathbf{R}\right) \\
& =J\left(-\alpha_{i m}^{-1} \alpha_{j m}^{-1} W_{i} W_{j} \hat{\phi}(\mathbf{W})\right) \\
& \equiv J \hat{\zeta}_{\mathrm{ex} 1}(\mathbf{W}),
\end{aligned}
$$

where $\hat{\phi}(\mathbf{W})$ is the Fourier representation of $\phi(\mathbf{R})$ and $\hat{\zeta}_{\mathrm{ex} 1}(\mathbf{W})$ is the Fourier representation of $\zeta_{\mathrm{ex} 1}(\mathbf{R}) \equiv$ $\left.f_{\mathrm{ex} 1}(\mathbf{r})\right|_{\mathbf{r}=\mathbf{R}}$. The above equation indicates that $\hat{\mathrm{e}}_{\mathrm{ex} 1}(\mathbf{w}(\mathbf{W}))$, or $\hat{\zeta}_{\text {ex } 1}(\mathbf{W})$, can be calculated from the (continuous) Fourier transform of $\phi(\mathbf{R})$. Since $\phi(\mathbf{R})$ is expressed with $\mathbf{R}$, the DFT can be implemented on the discrete $\phi(\mathbf{R})$ data since one can always choose to sample $\phi(\mathbf{R})$ along the directions of $\mathbf{e}_{i}$. Furthermore, it can be easily shown that for $f_{\text {ex } 1}=\nabla_{\mathbf{r}}^{2 n} \phi(\mathbf{R}(\mathbf{r}))$, where $n$ is a positive integer, one will have $\hat{\zeta}_{\mathrm{ex} 1}(\mathbf{W})=\left(-\alpha_{i m}^{-1} \alpha_{j m}^{-1} W_{i} W_{j}\right)^{n} \hat{\phi}(\mathbf{W})$.

\subsection{Inverse Transformation}

Here I derive the scheme for the inverse Fourier transform to obtain $f_{\text {ex } 1}(\mathbf{r})$. By using Eqs. (4) and (14), one writes

$$
\begin{aligned}
f_{\mathrm{ex} 1}(\mathbf{r}) & =\frac{1}{(2 \pi)^{d}} \int_{-\infty}^{\infty} \hat{f}_{\mathrm{ex} 1}(\mathbf{w}(\mathbf{W})) e^{i \mathbf{W} \cdot \mathbf{r}} d \mathbf{w} \\
& =\frac{J}{(2 \pi)^{d}} \int_{-\infty}^{\infty} \hat{\zeta}_{\mathrm{ex} 1}(\mathbf{W}) e^{i \mathbf{w} \cdot \mathbf{r}} d \mathbf{w},
\end{aligned}
$$

where the Fourier basis $e^{i \mathbf{w} \cdot \mathbf{r}}$ is used to maintain consistency with that used in Eq. (9). Next, one rewrites the integral with

$$
\int_{-\infty}^{\infty} d \mathbf{w}=\frac{1}{J} \int_{-\infty}^{\infty} d \mathbf{W}
$$

and modifies the Fourier basis as

$$
\begin{aligned}
\exp (i \mathbf{W} \cdot \mathbf{r}) & =\exp \left[i\left(\boldsymbol{\alpha}^{-T} \mathbf{W}\right) \cdot \mathbf{r}\right] \\
& =\exp \left[i \mathbf{W} \cdot\left(\boldsymbol{\alpha}^{-1} \mathbf{r}\right)\right] \\
& =\exp [i \mathbf{W} \cdot \mathbf{R}] .
\end{aligned}
$$

From Eqs. (16) and (17), Eq. (15) becomes

$$
\begin{aligned}
f_{\mathrm{ex} 1}(\mathbf{r}(\mathbf{R})) & =\frac{1}{(2 \pi)^{d}} \int_{-\infty}^{\infty} \hat{\zeta}_{\mathrm{ex} 1}(\mathbf{W}) e^{i \mathbf{W} \cdot \mathbf{R}} d \mathbf{W} \\
& =\zeta_{\mathrm{ex} 1}(\mathbf{R}) .
\end{aligned}
$$

Equation (18) indicates that when one performs the inverse DFT on the discrete $\hat{\zeta}_{\text {ex } 1}(\mathbf{W})$ data obtained from Section 3.1 , the result is the discrete values of $\zeta_{\operatorname{ex} 1}(\mathbf{R})$. Then one simply associates these data with the corresponding deformed coordinates $\mathbf{r}(\mathbf{R})$ to obtain the discretized $f_{\text {exl }}(\mathbf{r})$ data.

\section{Application to CDFT-Type PFC Free Energy}

A more general form of the PFC FED is that of the CDFTtype PFC model [4]:

$$
f_{\mathrm{pfc}}=\frac{\phi(\mathbf{x})}{2} \int_{-\infty}^{\infty} C\left(\mathbf{x}-\mathbf{x}^{\prime}\right) \phi\left(\mathbf{x}^{\prime}\right) d \mathbf{x}^{\prime}+\mathcal{L}(\phi(\mathbf{x})),
$$

where $\mathcal{L}(\phi(\mathbf{x}))$ is a function of terms with no derivative. The other part is the "non-local" term where the (nonlocal) operation is expressed in terms of a convolution of a two-body correlation function $C\left(\mathbf{x}-\mathbf{x}^{\prime}\right)$ and $\phi$. In the case of the FED in Eq. (2), one can see that $\mathcal{L}(\phi(\mathbf{x}))=\phi(\mathbf{x})^{4} / 4$ and $C\left(\mathbf{x}-\mathbf{x}^{\prime}\right)=\left[-\epsilon+\left(\nabla^{2}+1\right)^{2}\right] \delta\left(\mathbf{x}-\mathbf{x}^{\prime}\right)$. The latter gives $\hat{C}(\mathbf{s})$ (Fourier representation of $\left.C\left(\mathbf{x}-\mathbf{x}^{\prime}\right)\right)$ in the form of

$$
\hat{C}(\mathbf{s})=-\epsilon+\left(-|\mathbf{s}|^{2}+1\right)^{2} .
$$

Similar to Section 3, one can calculate the free energy of a deformed system from Eq. (6) where $f_{\text {def }}(\mathbf{r})$ is

$$
\begin{aligned}
f_{\mathrm{def}}(\mathbf{r})= & \frac{\phi\left(\boldsymbol{\alpha}^{-1} \mathbf{r}\right)}{2} \int_{-\infty}^{\infty} C\left(\mathbf{r}-\mathbf{r}^{\prime}\right) \phi\left(\boldsymbol{\alpha}^{-1} \mathbf{r}^{\prime}\right) d \mathbf{r}^{\prime} \\
& +\mathcal{L}\left[\phi\left(\boldsymbol{\alpha}^{-1} \mathbf{r}\right)\right] .
\end{aligned}
$$

To derive the FSM scheme, I consider a simplified example of

$$
f_{\mathrm{ex} 2}(\mathbf{r})=\int_{-\infty}^{\infty} C\left(\mathbf{r}-\mathbf{r}^{\prime}\right) \phi\left(\boldsymbol{\alpha}^{-1} \mathbf{r}^{\prime}\right) d \mathbf{r}^{\prime}
$$

As shown in Appendix A, the (continuous) Fourier transform of $f_{\mathrm{ex} 2}(\mathbf{r})$ is

$$
\hat{f}_{\mathrm{ex} 2}(\mathbf{w})=\hat{C}(\mathbf{w}) \int_{-\infty}^{\infty} e^{-i \mathbf{w} \cdot \mathbf{r}} \phi\left(\boldsymbol{\alpha}^{-1} \mathbf{r}\right) d \mathbf{r} .
$$

Using Eqs. (10) and (11), one arrives at

$$
\begin{aligned}
\hat{f}_{\mathrm{ex} 2}(\mathbf{w}(\mathbf{W})) & =J\left[\hat{C}\left(\boldsymbol{\alpha}^{-T} \mathbf{W}\right) \int_{-\infty}^{\infty} e^{-i \mathbf{W} \cdot \mathbf{R}} \phi(\mathbf{R}) d \mathbf{R}\right] \\
& =J\left[\hat{C}\left(\boldsymbol{\alpha}^{-T} \mathbf{W}\right) \hat{\phi}(\mathbf{W})\right] \\
& =J \hat{\zeta}_{\mathrm{ex} 2}(\mathbf{W}) .
\end{aligned}
$$

The above equation indicates that one can obtain $\hat{f}_{\text {ex } 2}\left(\mathbf{w}(\mathbf{W})\right.$ ), and also $\hat{\zeta}_{\text {ex } 2}(\mathbf{W})$, from the product of $\hat{\phi}(\mathbf{W})$ and $\hat{C}\left(\boldsymbol{\alpha}^{-T} \mathbf{W}\right)$. Since the two-body correlation function is typically specified in Fourier space, $\hat{C}\left(\alpha^{-T} \mathbf{W}\right)$ is simply obtained from functional evaluation at the (transformed) Fourier wave vector $\boldsymbol{\alpha}^{-T} \mathbf{W}$. From the above equation, the result from Section 3.1 can be obtained by recognizing that $\hat{C}(\mathbf{w})=-|\mathbf{w}|^{2}=-\mathbf{w} \cdot \mathbf{w}$ and, therefore, $\hat{C}\left(\alpha^{-T} \mathbf{W}\right)=$ $-\left[\boldsymbol{\alpha}^{-T} \mathbf{W}\right]_{m}\left[\boldsymbol{\alpha}^{-T} \mathbf{W}\right]_{m}=-\alpha_{i m}^{-1} W_{i} \alpha_{j m}^{-1} W_{j}$. This leads to $\hat{\zeta}_{\mathrm{ex} 2}(\mathbf{W})=-\alpha_{i m}^{-1} \alpha_{j m}^{-1} W_{i} W_{j} \hat{\phi}(\mathbf{W})=\hat{\zeta}_{\mathrm{ex} 1}(\mathbf{W})$, as expected. The procedure for the inverse Fourier transform is similar to that described in Section 3.2. 


\section{Numerical Verification}

The FSM scheme developed in this work will be verified by calculating $F_{\text {def }}$ (Eq. (6)) resulting from the deformation specified in Eq. (5). The order parameter profile is that of a body-centered cubic structure, $\phi(\mathbf{R})=\bar{\phi}+A\left[\cos \left(q R_{1}\right) \cos \left(q R_{2}\right)+\cos \left(q R_{1}\right) \cos \left(q R_{3}\right)+\right.$ $\left.\cos \left(q R_{2}\right) \cos \left(q R_{3}\right)\right]$, where $A$ is the amplitude, $\bar{\phi}$ is the average value and $q$ is the parameter that sets the scale of the structure. The parameters used are $A=1, \epsilon=0.15, \bar{\phi}=$ -0.23 , and $q=1 / \sqrt{2}$; the latter sets the lattice parameter $L=2 \sqrt{2} \pi$. The coordinates $R_{i}$ is discretized into $N=32$ intervals with the spacing $\Delta R_{i}=L / N$ and the coordinate values are $R_{i}=\Delta R_{i}[0,1,2, \ldots, N-1]$. The Fourier coordinates $\mathbf{W}$ will have the spacing $\Delta W_{i}=2 \pi / L$ and, since Matlab software is used, $W_{i}=\Delta W_{i}[0,1, \ldots, N / 2,-N / 2+$ $1,-N / 2+2, \ldots,-1]$. The DFT and the inverse DFT is performed using the "fftn" and "ifftn" functions, respectively, which employ a fast Fourier transform algorithm.

Figure 1 shows three values of $F_{\text {def }} / V$ as functions of $\xi$. The first result, labeled "Analytical", is the analytical solution obtained from symbolic computation. The second result, denoted "Laplacian", is the numerical calculation where the terms with derivatives in $f_{\text {def }}$ (Eqs. (7)) are calculated using the FSM scheme developed in Section 3. The third result, labeled "Correlation", is the numerical calculation using the FED from Eq. (19) with the two-body correlation function from Eq. (20). From the figure, one can see that the results from the three calculations agree with one another; the differences among these values are negligibly small. These results validate the numerical implementation of the FSM scheme proposed for both the original and CDFT-type PFC models.

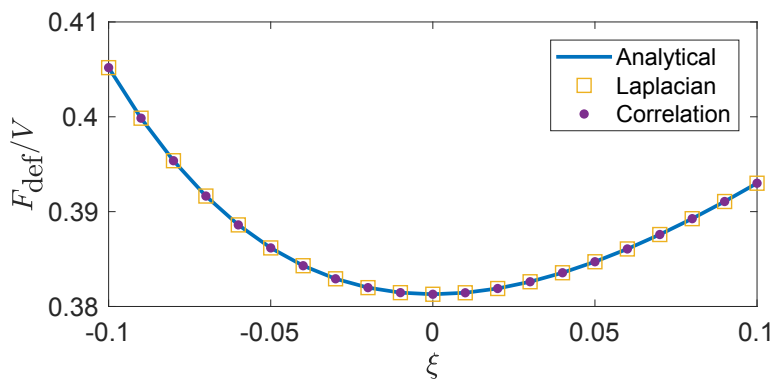

Figure 1. Plot of $F_{\text {def }} / V$ as a function of $\xi$. (See text for explanation.)

\section{Summary}

The FSM scheme for evaluating the PFC free energy of a system subjected to homogeneous deformation was developed. This scheme employs real-space coordinate transformation so that the (continuous) Fourier transform is performed on the function of the undeformed coordinates and the effect of the deformation is realized in the transformation of the Fourier wave vector. The advantage of this scheme is that it allows straightforward DFT implementation because the sampling at the undeformed configuration can be chosen to be along the directions of $\mathbf{e}_{i}$. This scheme can be used to efficiently and accurately evaluate the derivative of an order parameter and the convolution of an order-parameter and a two-body correlation function. In addition to the PFC model, this scheme applies to other models that can take advantages of the efficiency and accuracy of the FSM.

This work was supported by King Mongkut's Institute of Technology Ladkrabang Research Fund under Grant No. KREF046103, Thailand Research Fund (TRF) under Grant No. TRG5880008, and High Performance Computing Services from (Thailand) National Electronics and Computer Technology Center (NECTEC). The author would like to thank Dr. Victor W. L. Chan for helpful discussion and comments.

\section{A Fourier Transform of Convolution Integral}

The (continuous) Fourier transform of $f_{\mathrm{ex} 2}$ is derived in this section. Denoting $\Delta=\mathbf{r}-\mathbf{r}^{\prime}$, one can write

$$
\hat{f}_{\mathrm{ex} 2}=\int_{-\infty}^{\infty} e^{-i \mathbf{w} \cdot \mathbf{r}}\left[\int_{-\infty}^{\infty} C(\Delta) \phi\left(\boldsymbol{\alpha}^{-1} \mathbf{r}^{\prime}\right) d \mathbf{r}^{\prime}\right] d \mathbf{r} .
$$

Using the fact that $e^{-i \mathbf{w} \cdot \mathbf{r}}=e^{-i \mathbf{w} \cdot \Delta} e^{-i \mathbf{w} \cdot \mathbf{r}^{\prime}}$ and rearranging the order of integral, one arrives at

$$
\hat{f}_{\mathrm{ex} 2}=\int_{-\infty}^{\infty} e^{-i \mathbf{w} \cdot \mathbf{r}^{\prime}} \phi\left(\boldsymbol{\alpha}^{-1} \mathbf{r}^{\prime}\right)\left[\int_{-\infty}^{\infty} e^{-i \mathbf{w} \cdot \Delta} C(\Delta) d \mathbf{r}\right] d \mathbf{r}^{\prime}
$$

Since $\mathbf{r}^{\prime}$ is constant for the inner integral, $d \mathbf{r}=d \Delta$ and one can write

$$
\begin{aligned}
\hat{f}_{\mathrm{ex} 2} & =\int_{-\infty}^{\infty} e^{-i \mathbf{w} \cdot \mathbf{r}^{\prime}} \phi\left(\boldsymbol{\alpha}^{-1} \mathbf{r}^{\prime}\right)\left[\int_{-\infty}^{\infty} e^{-i \mathbf{w} \cdot \Delta} C(\Delta) d \Delta\right] d \mathbf{r}^{\prime} \\
& =\int_{-\infty}^{\infty} e^{-i \mathbf{w} \cdot \mathbf{r}^{\prime}} \phi\left(\boldsymbol{\alpha}^{-1} \mathbf{r}^{\prime}\right) \hat{C}(\mathbf{w}) d \mathbf{r}^{\prime}
\end{aligned}
$$

Changing the coordinates from $\mathbf{r}^{\prime}$ to $\mathbf{r}$, one obtains $\hat{f}_{\mathrm{ex} 2}=$ $\hat{C}(\mathbf{w}) \int_{-\infty}^{\infty} e^{-i \mathbf{w} \cdot \mathbf{r}} \phi\left(\boldsymbol{\alpha}^{-1} \mathbf{r}\right) d \mathbf{r}$, which is Eq. (23).

\section{References}

[1] K.R. Elder, M. Katakowski, M. Haataja, M. Grant, Phys. Rev. Lett. 88, 245701 (2002)

[2] H. Emmerich, H. Löwen, R. Wittkowski, T. Gruhn, G.I. Tóth, G. Tegze, L. Gránásy, Adv. Phys. 61, 665 (2012)

[3] G. Shechter, Ph.D. thesis, Johns Hopkins University (2004)

[4] K.R. Elder, N. Provatas, J. Berry, P. Stefanovic, M. Grant, Phys. Rev. B 75, 064107 (2007)

[5] K.R. Elder, M. Grant, Phys. Rev. E 70, 051605 (2004)

[6] K.A. Wu, A. Adland, A. Karma, Phys. Rev. E 81, $061601(2010)$ 